\title{
A preface to the international forum as a source of diversity opinions
}

\author{
Akio Koizumi
}

Published online: 3 February 2012

(C) The Japanese Society for Hygiene 2012

In this first 2012 issue of Environmental Health and Preventive Medicine (EHPM), I regrettably think back to the tragedy of the earthquake in north-east Japan and the Fukushima Daiichi Nuclear Power Plant accident. As president of our society, Dr. Hiroshi Sato issued a public statement representing the view of the board members that we had not been creative enough in science. Yes, creativity is important. But why public remark on 'deficiency' in creativity? I finally came up with my own answer: deficiency in generosity, curiosity and modesty. Deficiency in any of these three basic characters results in a vicious cycle that results in a deficiency in the other two characters. The authorities are not generous, curious and modest enough to listen to minority opinions because they are concerned about making concessions. I am now fully aware that many qualified scientists had warned of possible dangers prior to the earthquake. These warnings, however, were deliberately ignored by scientific authorities.
EHPM is rapidly becoming one of the major journals in the area of public health in Asian countries. Not only established and influential scientists but also young, upcoming leaders in various scientific fields are becoming members of the EHPM editorial board. To further foster the global development of EHPM, the Editorial Board has decided to add a section entitled "International Forum" to each issue of the journal. The aim of the forum is to expose readers to a wide diversity of opinions. Each forum article will be written by one or more of the members of the International Editorial Board. The first forum article is contributed by myself, Dr. Kyungho Choi and other Asian colleagues. The article reports on vividly evident current environmental health problems in Asian countries and raises several important agendas for the next 30 years. Therefore, we strongly believe that it is a worthy first article for the "International Forum".

\footnotetext{
A. Koizumi $(\square)$

School of Public Health, Graduate School of Medicine,

Kyoto University, Kyoto, Japan

e-mail: koizumi.akio.5v@kyoto-u.ac.jp
} 